\title{
PET and MRI in gliomas: progress and perspectives
}

\author{
Karl Herholz ${ }^{1}$
}

Received: 2 August 2017 / Accepted: 14 November 2017 / Published online: 1 January 2018

(c) Italian Association of Nuclear Medicine and Molecular Imaging 2017

A recent series of articles in this journal addressed the current status of brain tumour imaging with PET and advanced MRI methods [1-6]. They demonstrate that we now have highly effective instruments to assess brain tumours in individual patients with potential to improve treatment selection and ultimately outcome. However, in clinical practice, standard CT or MRI methods are still the main tools to diagnose tumours and to initiate treatment or further invasive diagnosis by biopsy. The current World Health Organisation brain tumour classification criteria [7] consider specific molecular markers for diagnosis. Unfortunately, none of them are currently accessible to imaging methods, but imaging techniques are essential for the evaluation of response to therapy. Advanced imaging techniques may overcome the limitations of standard contrast-enhanced CT or MRI on issues of pseudoresponse and pseudoprogression after therapy [8]. In particular, the amino acid tracers have been recognised by the European Association for Neuro-Oncology and the European Society for Medical Oncology as promising and widely applicable tools $[9,10]$.

Amino acid transporters are expressed in brain tissue and at the blood-brain barrier (BBB) to provide the essential amino acids needed for protein synthesis and other metabolic pathways. Their expression is increased in most tumours probably in correspondence with their metabolic needs. Although the initial studies were aiming explicitly at imaging of protein synthesis, subsequent studies using amino acid tracers not incorporated into proteins demonstrated that the increased signal in brain tumours mainly reflects increased amino acid transport [11]. Building on that legacy, superior signal to background and relatively low image noise with 18F-fluoroethyltyrosine (FET) PET compared to MR perfusion-weighted imaging is reported in a review by [2], supporting the use of amino acid PET for tailoring resection,

Karl Herholz

Karl.Herholz@manchester.ac.uk

1 Division of Neuroscience \& Experimental Psychology, University of Manchester, Manchester, UK guiding biopsies, assessment of tumour recurrence and progression, and planning of radiation therapy [3].

Identification of tumour hypoxia is an important issue, because it causes resistance to radiation effects. Most frequently $18 \mathrm{~F}$-fluoromisonidazole is being used, while 18F-FAZA provides an attractive alternative [12]. Promising results have been reported for detection of hypoxic tissue, but it is not yet clear whether adjustment of radiotherapy based on PET finding leads to better therapeutic outcomes [3].

In addition to amino acids, a broad range of other radiopharmaceuticals is being used and explored [13]. In this journal, the potential of choline, which can be labelled either by $11 \mathrm{C}$ or $18 \mathrm{~F}$ and is an essential constituent of cell membranes, is being presented [6]. The concept of assessing membrane turnover, which is increased in many tumours, has some similarity with the concept of assessing protein synthesis as a marker of cellular proliferation by amino acid tracers. It is already clear for amino acids that transport, not metabolism, is the critical issue that governs the image signal, and this also seems likely for the choline tracers.

Matching imaging and therapy targets is the goal of theranostics, which is an increasingly hot topic in oncology [14]. However, transport and metabolic processes usually cannot be used as direct therapeutic targets even when increased in tumours, because they are also essential in most other cells, and their global inhibition would cause severe side effects. Whether theranostics based on biomolecules or nanoparticles is a realistic goal in glioma is not yet clear, mainly because of the BBB that causes exclusion or very slow transfer rates for large molecules when intact, and non-specific uptake when broken. Potential targets include tumour growth factors that can possibly be imaged specifically, and recently, the mitochondrial translocator protein TSPO has been identified as a tumour marker related to proliferation that could also serve as a therapeutic target [1]. Also factors associated with angiogenesis are of direct interest for planning and assessing antiangiogenic therapy [15].

Interpretation of PET scans of brain tumours is difficult without reference to structural imaging methods. This is 
most obvious with FDG PET, where the contrast between tumour and brain is poor on PET scans because of the high and inhomogeneous uptake of FDG in brain. Thus, the quality of radiopharmaceuticals is sometimes judged by their tumour to background contrast, which clearly favours radiopharmaceuticals that do not cross the intact BBB and, therefore, have very little signal in normal brain. However, that concept is misleading, because very often radiopharmaceutical uptake in tumours then mainly reflects BBB damage, not providing much additional information beyond contrast-enhanced CT or MRI. In addition, such radiopharmaceuticals usually fail to provide a reliable signal in low-grade gliomas which still may have an intact blood-brain barrier, but may infiltrate intact brain and already be transforming to a higher grade.

Up-to-date provision of brain tumour PET scanning includes 3D coregistration with structural scans, usually contrast-enhanced and ideally even multimodal MRI. Thus, the location and extent of solid or contrast-enhancing tumour is determined by MR, and there is no need for a strong tumour-to-background contrast on PET. PET scans then can focus on molecular and metabolic characteristics of tumours, their infiltration zone, and remote but functionally important alterations in brain tissue, which is usually achieved most accurately with radiopharmaceuticals that show reasonably high extraction and some uptake also in normal brain tissue like FDG. While this can be done by PET-MR coregistration of separately acquired scans with standard software if scans were acquired with high resolution in all directions, it has become more convenient by hybrid PET/MR with simultaneous acquisition. Hybrid PET/MR also allows joint analysis of low-resolution MR signals, such as magnetic resonance spectroscopy [4]. However, there are also some technical challenges, especially with PET attenuation correction, and with integration into the standard clinical workflow that have not yet been resolved completely [5].

There has been huge progress with molecular PET and multimodal MR imaging methods, but many brain tumour PET papers published so far fail to compare imaging results with other molecular markers such as MGMT, IDH1, and EGFR mutations and $1 \mathrm{p} / 19 \mathrm{q}$ co-deletion [16], which are regarded as standard tools and may actually provide similar, complementary, or possibly even conflicting diagnostic and prognostic information [17]. Thus, future imaging studies need to compare results with these markers for the classification and management of brain tumours. We still will need further studies focussing on new imaging methods and radiopharmaceuticals with higher specificity, while also existing radiopharmaceuticals and multimodal imaging techniques should increasingly be applied in clinical trials and large prospective cohort studies to firmly establish their value as diagnostic, prognostic, and outcome markers.

\section{Compliance with ethical standards}

Conflict of interest The author declares that he has no conflict of interest.

Ethical approval This article does not contain any studies with human participants performed by any of the authors.

\section{References}

1. Roncaroli F, Su Z, Herholz K, Gerhard A, Turkheimer FE (2016) TSPO expression in brain tumours: is TSPO a target for brain tumour imaging? Clin Transl Imaging 4:145

2. Filss CP, Cicone F, Shah NJ, Galldiks N, Langen K-J (2017) Amino acid PET and MR perfusion imaging in brain tumours. Clin Transl Imaging 3(5):209-223

3. Oehlke O, Grosu A-L (2016) PET/MRI and brain tumors: focus on radiation oncology treatment planning. Clin Transl Imaging 2(5):159-167

4. Pyka T, Gempt J, Bette S, Ringel F, Förster S (2017) Positron emission tomography and magnetic resonance spectroscopy in cerebral gliomas. Clin Transl Imaging 5(2):151-158

5. Marner L, Henriksen OM, Lundemann M, Larsen VA, Law I (2017) Clinical PET/MRI in neurooncology: opportunities and challenges from a single-institution perspective. Clin Transl Imaging 5(2):135-149

6. Dardel NT, Gómez-Río M, Triviño-Ibáñez E, Llamas-Elvira JM (2016) Clinical applications of PET using C-11/F-18-choline in brain tumours: a systematic review. Clin Transl Imaging 2(5):101-119

7. Louis DN, Wiestler OD (eds) (2016) WHO classification of tumours of the central nervous system, 4th edn. International Agency for Research on Cancer, Lyon, France

8. Wen PY, Macdonald DR, Reardon DA, Cloughesy TF, Sorensen AG, Galanis E, Degroot J, Wick W, Gilbert MR, Lassman AB et al (2010) Updated response assessment criteria for high-grade gliomas: response assessment in neuro-oncology working group. J Clin Oncol 28:1963-1972

9. Stupp R, Tonn JC, Brada M, Pentheroudakis G (2010) High-grade malignant glioma: ESMO Clinical Practice Guidelines for diagnosis, treatment and follow-up. Ann Oncol 21(suppl_5):v190-v193

10. Weller M, van den Bent M, Tonn JC, Stupp R, Preusser M, CohenJonathan-Moyal E, Henriksson R, Le Rhun E, Balana C, Chinot O et al (2017) European Association for Neuro-Oncology (EANO) guideline on the diagnosis and treatment of adult astrocytic and oligodendroglial gliomas. Lancet Oncol 18(6):e315-e329

11. Wienhard K, Herholz K, Coenen HH, Rudolf J, Kling P, Stocklin G, Heiss WD (1991) Increased amino acid transport into brain tumors measured by PET of L-(2-18F)fluorotyrosine. J Nucl Med 32(7):1338-1346

12. Mapelli P, Incerti E, Bettinardi V, Conte GM, Fallanca F, Bailo M, Vuozzo M, Callea M, Gianolli L, Picchio M (2017) Hypoxia 18F-FAZA PET/CT imaging in lung cancer and high-grade glioma: open issues in clinical application. Clin Transl Imaging 4(5):389-397

13. Herholz K (2017) Brain tumors: an update on clinical PET research in gliomas. Semin Nucl Med 47(1):5-17

14. Jacobson O, Chen X (2013) Interrogating tumor metabolism and tumor microenvironments using molecular positron emission tomography imaging. Theranostic approaches to improve therapeutics. Pharmacol Rev 65(4):1214-1256

15. Iagaru A, Mosci C, Mittra E, Zaharchuk G, Fischbein N, Harsh G, Li G, Nagpal S, Recht L, Gambhir SS (2015) Glioblastoma 
multiforme recurrence: an exploratory study of 18F FPPRGD2 PET/CT. Radiology 277(2):497-506

16. Siegal T (2015) Clinical impact of molecular biomarkers in gliomas. J Clin Neurosci 22(3):437-444

17. Verger A, Metellus P, Sala Q, Colin C, Bialecki E, Taieb D, Chinot O, Figarella-Branger D, Guedj E (2017) IDH mutation is paradoxically associated with higher 18F-FDOPA PET uptake in diffuse grade II and grade III gliomas. Eur J Nucl Med Mol I 44(8):1306-1311 